\title{
Rotational Spectrum of Methylcyanoacetylene A New Millimeter Wave Spectrometer
}

\author{
M. Bester, M. Tanimoto*, B. Vowinkel, G. Winnewisser, and Koichi Yamada \\ I. Physikalisches Institut, Universität zu Köln
}

Z. Naturforschung, 38 a, 64-67 (1983); received October 28, 1982

\begin{abstract}
The ground state rotational spectrum of methylcyanoacetylene, $\mathrm{CH}_{3} \mathrm{CCCN}$, has been observed between 8 and $90 \mathrm{GHz}$, yielding precise rotational constants and a determination of the nuclear hyperfine costant $e q Q=-4.0 \pm 0.2 \mathrm{MHz}$. The millimeter wave spectra $(70-90 \mathrm{GHz})$ were obtained with a newly constructed spectrometer, employing a synthesizer - controlled reflex klystron as source.
\end{abstract}

\section{Indroduction}

Methylcyanoacetylene, $\mathrm{CH}_{3} \mathrm{CCCN}$, as a member of the unsaturated carbon chain molecules could be of potential astrophysical interest. Being related to the abundant interstellar molecule $\mathrm{HCCCN}$, it bears similar chemical characteristics, in particular that it also can be generated in a radio-frequency discharge burning in a methylacetylene $\left(\mathrm{CH}_{3} \mathrm{CCH}\right)$ /hydrogen cyanide $(\mathrm{HCN})$ gas mixture as has been demonstrated earlier [1]. The present new data, however, have been obtained from a chemically prepared sample**.

Although the ground state microwave spectrum of methylcyanoacetylene has been reported as early as 1954 by Sheridan and Thomas [2], there is no further mention of this molecule in the literature until the recent discharge experiments [1] and now the publication of the ground state millimeter wave spectrum of this molecule by Moïses et al. [3]. However several aspects of the rotational spectrum of $\mathrm{CH}_{3} \mathrm{CCCN}$ have not yet been treated with the accuracy desired for astrophysical purposes: there is no reliable determination of the ${ }^{14} \mathrm{~N}$-nuclear quadrupole constant, several ground state transitions at low and medium $J$ values have not been measured and finally there exist no measurements of rotational transitions in vibrationally excited bending states.

The purpose of the present contribution is twofold: i) to report supplemental measurements on the

* On leave from Sagami Chemical Research Center, Nishi-Ohnuma 4-4-1, Sagamihara, Kanagawa 229, Japan.

** Kindly given to us from Dr. J. Demaison of the Laboratoire de Spectroscopie Hertzienne, Université de Lille, France.

Reprint requests to M. Bester, I. Physikalisches Institut, Universität zu Köln, 5000 Köln. grond state of $\mathrm{CH}_{3} \mathrm{CCCN}$, which have been carried out with a newly constructed millimeter wave spectrometer and ii) to discuss briefly the essential design features of the Cologne millimeter wave spectrometer employed in the present measurements. Although in the present measurements many lines arising from the molecules in excited bending vibrational states have been observed, both in the millimeter and microwave region, they will not be reported here, pending their detailed analysis.

\section{Measurements}

The measurement of several new millimeter wave transitions were complemented by observation of rotational transitions in the microwave region, particularly to obtain a reliable determination of the ${ }^{14} \mathrm{~N}$-nuclear quadrupole coupling constant, which is of importance to the possible identification of narrow interstellar $\mathrm{CH}_{3} \mathrm{CCCN}$ lines at low and medium $J$ values, arising from dark clouds - such as TMC1.

The measured $\mathrm{CH}_{3} \mathrm{CCCN}$ transitions at low $J$ are remarkably broad, probably caused by the combined effects of $K$-structure, nuclear hyperfine structure and contribution from the remaining pressure broadening due to the large dipole moment. Therefore only the observed profile of the $J=2-1$ transition could be used for a reliable $e q Q$ determination. Our new data are summarized in Table 1.

\section{Millimeter wave spectrometer}

The new Cologne millimeter wave spectrometer is of the free space type and is similar to the one in Giessen discussed by Winnewisser et al. [4] and 
Table 1. Observed Transitions and Fitted Parameters.

\begin{tabular}{llll}
\hline$(J+1, K)-(J, K)$ & $f_{\text {obs }} / \mathrm{MHz}$ & $f_{\text {calc }} / \mathrm{MHz}$ & $\begin{array}{l}f_{\text {obs }}-f_{\text {calc }} / \\
\mathrm{kHz}\end{array}$
\end{tabular}

\begin{tabular}{|c|c|c|c|}
\hline 0$)-(1,0)$ & $8263.000^{\mathrm{a}}$ & 8262.9505 & 49.5 \\
\hline 0$)-(2$ & $12394.360^{\mathrm{b}}$ & 12394.4201 & 60.1 \\
\hline 0$)-(4$, & $20657.325^{\mathrm{b}}$ & 20657.3369 & 11.9 \\
\hline 0$)-(5$ & $24788.200^{c}$ & 24788.7795 & -579.5 \\
\hline 0$)-(17$ & 74364.385 & 74364.3986 & -13.6 \\
\hline$(18,1)-(17,1)$ & 74363.658 & 74363.6823 & 24.3 \\
\hline$(18,2)-(17,2)$ & 74361.516 & 74361.5333 & 17.3 \\
\hline$(18,3)-(17$ & 74357.918 & 74357.9522 & 34.2 \\
\hline 4) - (17, & 74352.922 & 74352.9398 & 17.8 \\
\hline 5$)-(17$ & 74346.462 & 74346.4972 & 35.2 \\
\hline 6$)-(17$ & 74338.563 & 74338.6258 & -62.8 \\
\hline 7) $-(17$ & 74329.322 & 74329.3273 & 5.3 \\
\hline$(18,8)-(17,8)$ & 74318.571 & 74318.6038 & 32.8 \\
\hline$(18,9)-(17,9)$ & 74306.480 & 74306.4576 & 22.4 \\
\hline$(18,10)-(17,10)$ & 74292.936 & 74292.8915 & 44.5 \\
\hline$(20,0)-(19,0)$ & 82626.518 & 82626.5408 & 22.8 \\
\hline 1) $-(19$ & 82625.760 & 82625.7450 & 15.0 \\
\hline 2$)-(19$ & 82623.380 & 82623.3580 & 22.0 \\
\hline 3) $-(19$, & 82619.393 & 82619.3802 & 12.8 \\
\hline$(20,4)-(19$, & 82613.790 & 82613.8124 & 22.4 \\
\hline$(20,5)-(19$ & 82606.740 & 82606.6560 & 84.0 \\
\hline 6$)-(19$ & 82597.987 & 82597.9125 & 74.5 \\
\hline 7) $-(19$ & 82587.620 & 82587.5839 & 36.1 \\
\hline 8$)-(19$ & 82575.645 & 82575.6723 & 27.3 \\
\hline (20, & 82562.150 & 82562.1805 & 30.5 \\
\hline & 57.524 & 757.5456 & 21.6 \\
\hline$(21,1)-(20,1)$ & 86756.698 & 86756.7102 & 12.2 \\
\hline$(21,2)-(20,2)$ & 86754.171 & 6754.2042 & -33.2 \\
\hline 3$)-(20$ & 86750.040 & 6750.0281 & 11.9 \\
\hline$(21,4)-(20,4)$ & 86744.220 & 86744.1829 & 37.1 \\
\hline 5$)-(20$ & 86736.666 & 86736.6699 & 3.9 \\
\hline & 727.490 & 86727.4907 & 0.7 \\
\hline$(21,7)-(20,7)$ & 716.618 & 86716.6473 & -29.3 \\
\hline$(21,8)-(20,8)$ & 86704.155 & 86704.1421 & 12.9 \\
\hline$(21,9)-(20,9)$ & 86689.970 & 86689.9779 & 7.9 \\
\hline$(21,10)-(20,10)$ & 86674.125 & 86674.1578 & -32.8 \\
\hline
\end{tabular}

a Line center is obtained by analyzing the hyperfine splittings. The $K=0$ and 1 lines are overlapping each other.

b Higher $K$ lines are overlapping. 25 times less weighted.

c Higher $K$ lines are overlapping. Not used in the fit. Number of lines fitted: 100 , Standard deviation: $35.0 \mathrm{kHz}$.

Fitted parameters:

$\begin{array}{llll}B=2065.73836 & (18) \mathrm{MHz} & H_{K J}=0.365 & (11) \mathrm{Hz} \\ D_{J K}=19.9242 & (28) \mathrm{kHz} & H_{J K}=0.03793 & (32) \mathrm{Hz} \\ D_{J}=93.550 & (56) \mathrm{Hz} & H_{J}=-0.0000087 & (61) \mathrm{Hz}\end{array}$

Helms et al. [5]. The particular difference between the Cologne spectrometer and the Giessen spectrometer lies in the frequency control system of the reflex klystron. Whereas the Giessen spectrometer employs a special sweep stabilizer to control the free running reflex klystron, the present system (Fig. 1) employs a phase-locked millimeter wave klystron, the frequency of which is controlled by a digital synthesizer, both in the video- and in the lock-in-mode (source frequency modulation with a sine wave and detection with a lock-in-amplifier). This phasestabilized klystron output is fed into the free space absorption cell via a corrugated horn antenna and a set of teflon lenses to collimate the beam. The glass cell has a length of about $1.8 \mathrm{~m}$ and a diameter of $10 \mathrm{~cm}$. The signal received by a detector horn is demodulated in a wave guide detector with following lock-in-amplifier. A microprocessor system controls the sweep and generates frequency markers on the dual pen recorder.

The phase-lock system is identical to the one which will be used in the millimeter wave receivers of the Cologne $3 \mathrm{~m}$ - millimeter wave radiotelescope, which is presently under construction at our institute [6]. The block diagram of the PLL (phase-locked loop) is presented in Figure 2. The reflex klystron is phase-locked by adding to the reflector voltage a correction voltage being proportional to the phase and frequency error of the klystron signal. A small part of the klystron output power is mixed with the n-th harmonic of a highly stabilized frequency synthesizer to obtain an IF-signal near $400 \mathrm{MHz}$, which contains a possible phase or frequency drift of the klystron. The IF-signal is levelled to a constant amplitude and then multiplied in a phase- and frequency-difference detector with a second $400 \mathrm{MHz}$ reference signal, derived from another frequency synthesizer in the lock-in-mode or a sweeper in the video-mode. The resulting phase- and frequency-difference signals are used to correct the klystron reflector voltage. The servo amplifier in the reflector line consists of two paths: i) the integral path which is coupled to the klystron reflector circuitry by an opto-coupler and ii) the fast proportional path linked by a capacitor. The coarse adjustment of the klystron frequency is done by the slow integral path, whereas the fine adjustment i.e. the phase-locking is done by the proportional path. If the frequency-difference signal is zero in the locked mode, the phase-difference signal dominates in the servo loop and provides continuous locking of the klystron to the $400 \mathrm{MHz}$ reference source.

By sweeping the frequency-modulated reference source (lock-in-mode) at $400 \mathrm{MHz}$ by an amount of $\pm 50 \mathrm{MHz}$, the klystron will follow instantaneously and its frequency is known exactly at any time as the center frequency of the modulated signal. The videomode $(10 \mathrm{~ms} / \mathrm{sweep})$ is presently used for adjusting the spectrometer. 


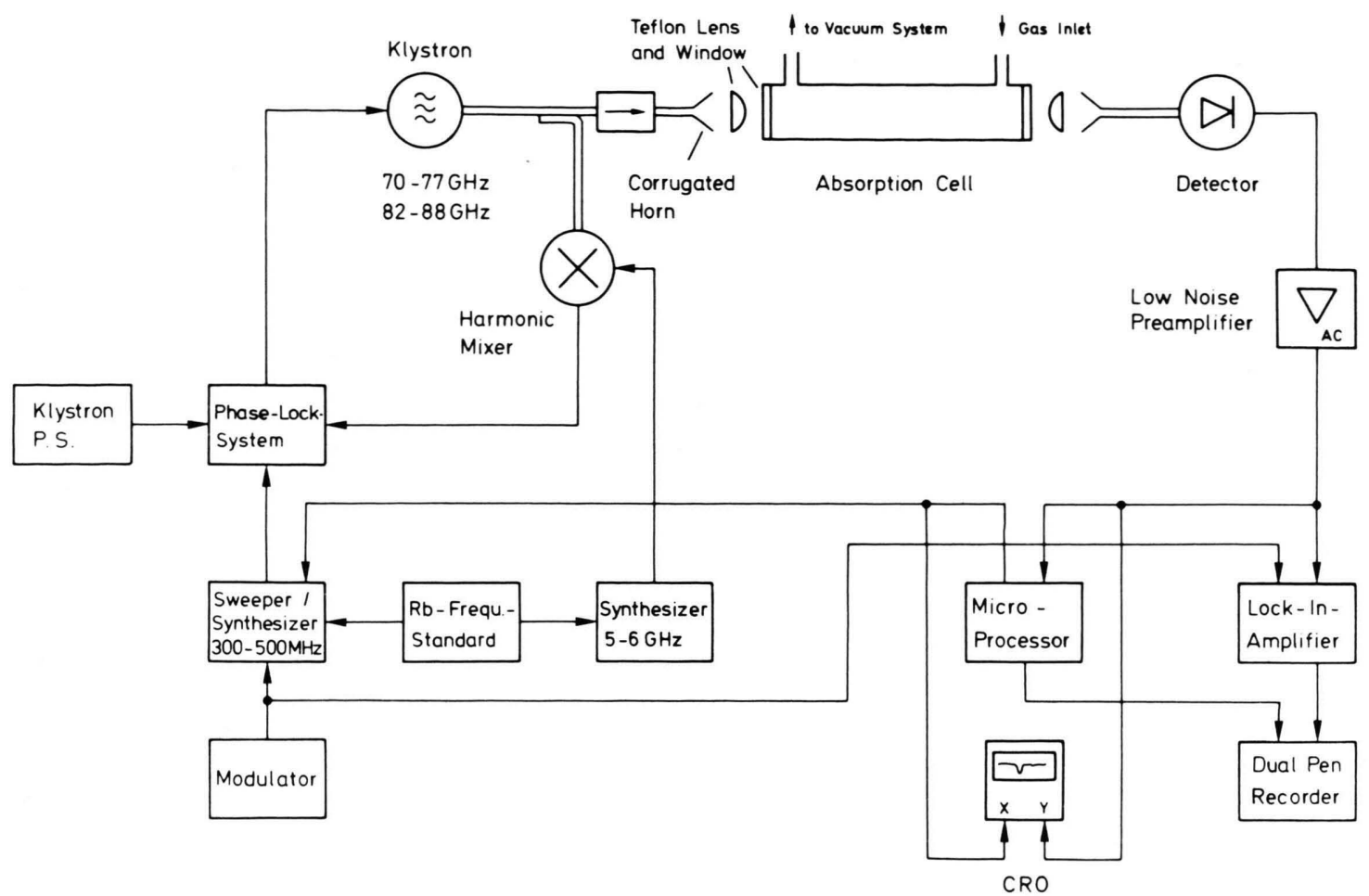

Fig. 1. Block diagram of the millimeter wave spectrometer. The spectrometer is of the free space type. The mm-wave sig$\mathrm{nal}$ is frequency - modulated and detected at the second harmonic of the modulation frequency.

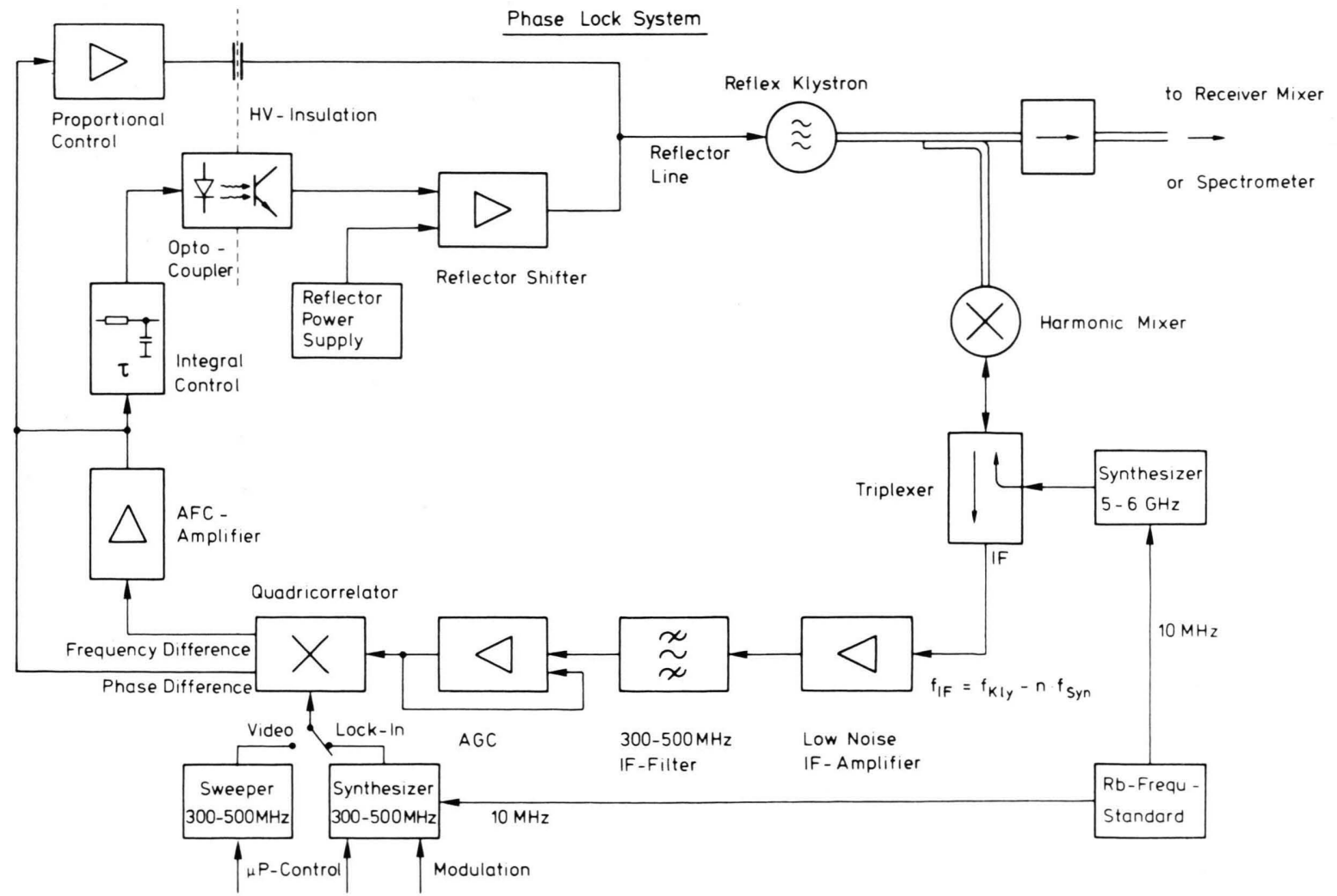

Fig. 2. Schematic diagram of the phase-locked loop (PLL). The PLL locks a reflex klystron to a stable reference signal. The system is also used as local oscillator in the receivers of the Cologne $3 \mathrm{~m}-$ millimeter radiotelescope. 


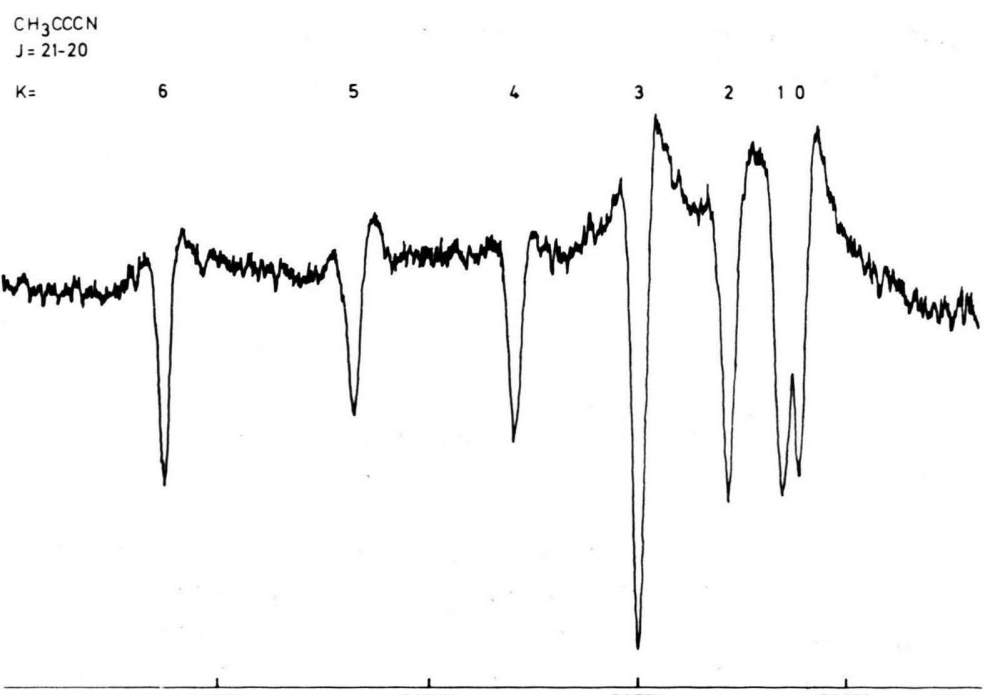

Fig. 3. Rotational spectrum of $\mathrm{CH}_{3}$ $\mathrm{CCCN}$ at $86 \mathrm{GHz}$. The spectrum has been measured in the lock-in-mode, sweeping the klystron with a rate of $100 \mathrm{kHz} / \mathrm{s}$.

The millimeter wave spectrometer will be further improved in due course by use of a frequency multiplier and a He-cooled InSb-detector to allow measurements in the submillimeter wave region. A microprocessor program for signal averaging in the video-mode will improve the sensitivity.

\section{Results and discussion}

The mm-wave-data presented in Table 1 and the recording shown in Fig. 3 were obtained in the lockin-mode, whereas the lines between 8 and $26 \mathrm{GHz}$ have been measured with the Stark-modulation Hewlett-Packard spectrometer [7], located at the University of Giessen.

The observed data have been fitted to the standard expression for rotational transition frequencies of symmetric rotors including the $\mathrm{D}$ - and $\mathrm{H}$-centrifugal distortion terms. The molecular parameters thus derived are listed in Table 1. For astrophysical reference the quoted constants are reported with suf- ficient significant digits to allow the calculated spectra to reproduce the best fit to the Moïses et al. [3] and our data.

The value for the ${ }^{14} \mathrm{~N}$-nuclear quadrupole constant has been obtained by fitting the position of the 10 strongest hyperfine components (see [8]) to the observed profile of the $J=2-1$ transition. The result is: $e q Q=-4.0 \pm 0.2 \mathrm{MHz}$

It may be noticed that the frequency of the $J=21-20, K=3$ line of $\mathrm{CH}_{3} \mathrm{CCCN}$ at $86.750 \mathrm{GHz}$ coincides within the error limits to one of the published unidentified interstellar lines [9]. A possible identification would require the interstellar detection of other lines of $\mathrm{CH}_{3} \mathrm{CCCN}$, which are now precisely known.

\section{Acknowledgements}

One of us (M.T.) expresses his gratitude for a Heinrich Hertz guest scientist fellowship. The work was supported in part by the Deutsche Forschungsgemeinschaft - SFB-131 - Radioastronomie.
[1] G. Winnewisser, F. Toelle, H. Ungerechts, and C. M. Walmsley, in IAU Sympos. No. 87, (B. H. Andrew ed.), Interstellar Molecules, (1980), pp. 59-65.

[2] J. Sheridan and L. F. Thomas, Nature London 174, 798 (1954).

[3] A. Moïses, D. Boucher, J. Burie, J. Demaison, and A. Dubrulle, J. Mol. Spectrosc. 92, 497 (1982).

[4] M. Winnewisser and B. P. Winnewisser, Z. Naturforsch. 29 a, 633 (1974)

[5] D. A. Helms, M. Winnewisser, and G. Winnewisser, J. Phys. Chem. 84, 1758 (1980).
[6] G. Winnewisser, M. Bester, E. Pofahl, M. Olberg, G. Rau, W. Zensen, P. Wratil, and B. Vowinkel, Proceedings of a Symposium on The Scientific Importance of Submillimetre Observations, ESA SP-189, 53 (1982).

[7] W. H. Hocking and G. Winnewisser, Z. Naturforsch. 31 a, 422 (1976).

[8] C. H. Townes and A. L. Schawlow, Microwave Spectroscopy, p. 154 Dover Publications Inc., New York 1975.

[9] A. H. Cook, Phil. Trans. Roy. Soc. London A 303, 551 (1981). 